Arq. Bras. Med. Vet. Zootec., v.70, n.3, p.850-856, 2018

\title{
Resveratrol atenua o estresse oxidativo e a lesão muscular de ratos sedentários submetidos ao exercício físico
}

[Resveratrol attenuates oxidative stress and muscle damage in sedentary rats subjected to physical exercise]

\author{
L.G. Narciso ${ }^{1}$, B.F.M. Almeida ${ }^{1}$, A.M. Bosco ${ }^{1}$, P.P. Pereira ${ }^{2}$, K.E. Vendrame ${ }^{1}$, \\ M.J.Q. Louzada ${ }^{3}$, P.C. Ciarlini ${ }^{3}$ \\ ${ }^{1}$ Aluno de pós-graduação - Unesp - Araçatuba, SP \\ ${ }^{2}$ Aluna de pós-graduação - Unesp - Jaboticabal, SP \\ ${ }^{3}$ Universidade Estadual Paulista "Júlio de Mesquita Filho" - Araçatuba, SP
}

\begin{abstract}
RESUMO
O sedentarismo é um problema de saúde pública e um dos maiores males da sociedade moderna. Já está bem estabelecido que esforço físico em excesso ou em indivíduos não condicionados acarreta estresse oxidativo e lesões musculares. No presente estudo, foi testada a hipótese de que um único esforço físico é capaz de causar estresse oxidativo e lesão muscular em indivíduos sedentários. Aditivamente foi avaliado efeito antioxidante do polifenol resveratrol (RV) quanto a sua capacidade de atenuar o estresse oxidativo e a lesão muscular causados pelo esforço físico. Para tal, 40 ratos (Rattus norvegicus albinus, Wistar), machos, adultos e sedentários foram aleatoriamente submetidos ou não a 90 minutos de natação, com e sem tratamento com RV (100mg/kg/PV/14dias): N-RV- (n=10) grupo mantido em repouso e não tratado com RV; $\mathrm{N}-\mathrm{RV}+(\mathrm{n}=10)$ grupo mantido em repouso e tratado com $\mathrm{RV}$; $\mathrm{N}+\mathrm{RV}$ - $(\mathrm{n}=10)$ grupo submetido ao esforço físico de natação e não tratado com $\mathrm{RV}$ e $\mathrm{N}+\mathrm{RV}+(\mathrm{n}=10)$ grupo submetido ao esforço físico de natação e tratado com $\mathrm{RV}$. Em ratos sedentários, o esforço físico da natação promoveu estresse oxidativo (aumento da peroxidação lipídica e diminuição da capacidade antioxidante total do plasma) e aumento significativo da atividade plasmática de creatina quinase (CK) e lactato desidrogenase (LDH). O tratamento com RV diminuiu a peroxidação lipídica e a concentração dos marcadores de lesão muscular $(\mathrm{CK}$ e LDH) de ratos sedentários submetidos à natação. Essa é uma das primeiras evidências de que um único esforço físico pode causar estresse oxidativo em indivíduos sedentários e que o RV pode ser uma alternativa para atenuar a lesão muscular causada por esse estresse.
\end{abstract}

Palavras-chave: antioxidante, natação, sedentarismo

\begin{abstract}
Physical inactivity is a public health problem when a sedentary population practices physical activity sporadically. Exercise in unconditioned individuals causes oxidative stress and muscle damage. This study tested the hypothesis that a single physical exertion can cause muscle damage and oxidative stress in sedentary individuals, and resveratrol can attenuate it. For this, 40 sedentary adult male rats were equally and randomized into four groups subjected to 90min swimming or rest and administered aqueous resveratrol $(100 \mathrm{mg} / \mathrm{kg} /$ day) or saline for 14 days: $N-R V$-, rats maintained at rest and administered saline; $N-R V+$, rats maintained at rest and treated with resveratrol; $N+R V$-, rats subjected to physical exercise and administered saline; and $N+R V+$, rats subjected to physical exercise and treated with resveratrol. In sedentary rats, the physical exertion of swimming promoted oxidative stress, i.e. increased lipid peroxidation and decreased plasma total antioxidant capacity, and significant increases in CK and LDH plasma activities. Resveratrol diminished lipid peroxidation and the concentrations of muscle damage markers (CK and LDH) in sedentary rats subjected to swimming. The results provide evidence that a single sudden physical exertion can cause oxidative stress in sedentary rats. Resveratrol showed good results as a treatment for minimizing muscle damage caused by this stress.
\end{abstract}

Keywords: oxidative stress, antioxidant treatment, physical exertion, physical inactivity

Recebido em 21 de outubro de 2016

Aceito em 7 de agosto de 2017

E-mail: luisgustavo.medvet@hotmail.com 


\section{INTRODUÇÃO}

A associação entre exercício físico e saúde vem se consolidando nos últimos anos. Estudos revelam que a prática regular de exercício físico está associada à promoção da saúde e à prevenção de doenças crônico-degenerativas (Bousquete-Santos et al., 2006).

O estresse oxidativo é um desequilíbrio entre as espécies reativas de oxigênio (ERO), em que o superóxido, o peróxido de hidrogênio e o radical hidroxil se sobrepõem às defesas antioxidantes (Powers; Jackson, 2008).

O exercício físico exaustivo ocasiona um desequilíbrio na homeostase intracelular entre os agentes pró e antioxidantes, levando ao aumento de ERO gerado pelo maior consumo de oxigênio (Deaton; Marlin, 2003). O estresse oxidativo decorrente de uma sessão de exercício físico pode causar danos a lipídeos, a proteínas e ao DNA, assim como lesões de fibras musculares, dor e inflamação (Deaton; Marlin, 2003). A intensidade do dano oxidativo e a elevação da atividade plasmática da creatina quinase (CK), do aspartato aminotransferase (AST), da alanina aminotransferase (ALT) e do lactato desidrogenase (LDH) dependem da intensidade, da duração, do ambiente e da resistência à exaustão muscular (Nie et al., 2010).

$\mathrm{O}$ uso de antioxidantes para prevenir o estresse oxidativo causado pelo exercício físico tem ganhado destaque. Há evidências de que o uso de suplementos antioxidantes, como as vitaminas $\mathrm{E}$ e C, o betacaroteno, a coenzima Q10, a Nacetilcisteína e o ácido úrico, podem minimizar ou até mesmo evitar os danos oxidativos musculares e plasmáticos induzidos pelo esforço físico moderado a intenso (Fischer-Wellman; Bloomer, 2009).

O resveratrol (RV) é um polifenol presente principalmente nas uvas, nos vinhos e nos amendoins, sendo capaz de promover proteção contra doenças metabólicas, melhorando a função e a biogênese mitocondrial (Baur; Sinclair, 2006). Há evidências também de que o $\mathrm{RV}$ previne a oxidação lipídica, aumenta a atividade das enzimas antioxidantes, melhora o desempenho muscular, altera o catabolismo proteico, além de evitar danos e apoptose de células do músculo esquelético durante o exercício físico em camundongos (Ryan et al, 2010).

Estudos experimentais sobre estresse oxidativo induzido pelo esforço físico realizados com ratos têm sido considerados mais confiáveis quando comparados aos realizados em humanos, uma vez que permitem minimizar diversos fatores que podem influenciar parâmetros utilizados para avaliarem a lesão muscular, o metabolismo oxidativo e o sistema de defesa antioxidante (Fischer-Wellman; Bloomer, 2009). Entretanto, são poucos os estudos que investigaram o efeito antioxidante do RV sobre o estresse oxidativo decorrente do esforço físico em ratos. Quando associado a outros antioxidantes, o RV foi capaz de reduzir o estresse oxidativo do esforço físico em ratos (Sun et al., 2011).

Nesse sentido, foi investigada a hipótese de que o RV é capaz de atenuar o estresse oxidativo e a lesão muscular de ratos sedentários submetidos a esforço físico.

\section{MATERIAL E MÉTODOS}

Trata-se de uma pesquisa feita inteiramente de modo aleatório, de tal forma que todos os animais tiveram a mesma oportunidade de serem sorteados para qualquer um dos quatro grupos e suplementados com o resveratrol, sendo realizada com a aprovação da Comissão de Ética no Uso de Animais da Unesp, Campus de Araçatuba (protocolo FOA-0568/11), de acordo com os princípios éticos na experimentação animal da Universidade Estadual Paulista "Júlio de Mesquita Filho", Campus de Araçatuba.

Foram utilizados 40 ratos (Rattus norvegicus albinus, Wistar), provenientes do biotério da Faculdade de Medicina Veterinária e Zootecnia de Botucatu-SP, todos machos, com 90 dias, com aproximadamente 350 gramas. Os animais foram mantidos em ambiente climatizado $\left(22 \pm 2^{\circ} \mathrm{C}\right)$, com ciclo claro/escuro (12/12 horas diárias), no Laboratório de Biofísica da Unesp, e tiveram um período de adaptação de 60 dias. Foram distribuídos em caixas comuns de polipropileno medindo $41 \times 34 \times 16$, com quatro animais por caixa, alimentados com ração própria para ratos $\left(\right.$ Purina $\left.{ }^{\circledR}\right)$ e água à vontade.

Todos os ratos, depois de numerados, foram sorteados, a fim de serem aleatoriamente 
submetidos a quatro tratamentos: grupo N-R$(n=10)$, constituído de ratos em repouso e tratados via oral por gavagem com $1 \mathrm{~mL}$ de solução aquosa de $\mathrm{NaCl} 0,9 \%$ por 14 dias; grupo $\mathrm{N}-\mathrm{R}+(\mathrm{n}=10)$, constituído de ratos em repouso e tratados via oral por gavagem com $1 \mathrm{~mL}$ de solução aquosa de RV (100mg/kgPV/14dias); grupo $\mathrm{N}+\mathrm{R}-\quad(\mathrm{n}=10)$, constituído de ratos submetidos ao esforço físico de natação e tratados via oral por gavagem com $1 \mathrm{~mL}$ de solução aquosa de $\mathrm{NaCl} 0,9 \%$ por 14 dias; e grupo $\mathrm{N}+\mathrm{R}+\quad(\mathrm{n}=10)$, constituído de ratos submetidos ao esforço físico de natação e tratados via oral por gavagem com $1 \mathrm{~mL}$ de solução aquosa de RV (100mg/kgPV/14dias). Para tal, utilizou-se RV comercial (Terraternal com $99 \%$ de pureza), e o protocolo de exercício físico de 14 dias utilizado no experimento foi segundo estudos realizados em ratos por Ikizler et al. (2006). A dose de $100 \mathrm{mg} / \mathrm{kg} / \mathrm{PV}$ foi utilizada segundo Pearson et al. (2008), os quais observaram bons resultados com essa concentração de resveratrol, sem causar alterações hepáticas e renais nos animais, sendo a última dose administrada 30 minutos antes do esforço físico. Foi utilizada a natação, a fim de evitar qualquer tipo de contusão.

A lesão muscular por estresse oxidativo foi induzida conforme protocolo de Lee et al. (2009), com pequenas modificações. Resumidamente, após o $14^{\circ}$ dia experimental, todos os ratos dos grupos $\mathrm{N}+\mathrm{R}-\mathrm{e} \mathrm{N}+\mathrm{R}+$ foram submetidos a uma única sessão de 90 minutos de natação, no mesmo horário, realizada no início da manhã durante todo o andamento do projeto. Para tal, os ratos foram colocados individualmente em um tanque de 60 centímetros de altura e 20 de diâmetro, com $55 \mathrm{~cm}$ de água, a qual foi mantida na temperatura de $31^{\circ} \pm 1^{\circ} \mathrm{C}$, sendo a atividade de natação continuamente monitorada quanto ao desconforto e à exaustão, conforme critérios descritos por Veskoukis et al. (2009)

Para a coleta de sangue dos ratos, estes foram anestesiados com cloridrato xilazina $2 \%$ $(3 \mathrm{mg} / \mathrm{kg})$ e cloridrato de cetamina $(30 \mathrm{mg} / \mathrm{kg})$ para punção cardíaca de $5 \mathrm{~mL}$ de sangue total, sendo as amostras armazenadas em frascos heparinizados protegidos da luz $(10 \mathrm{UI} / \mathrm{mL})$ para imediata obtenção do plasma por centrifugação $(3600 \mathrm{~g} / 10$ minutos) e guardadas em caixas de papel até o momento das análises laboratoriais.
As amostras de sangue total dos ratos em repouso foram coletadas juntamente com as dos ratos submetidos à natação. A eutanásia foi realizada com dose 10 vezes maior à citada anteriormente de cloridrato de xilazina $2 \%$ e cloridrato de cetamina.

Para tal, foi realizada a dosagem de colesterol e a de triglicerídeos. A fim de evitar interferência da hemólise, os biomarcadores plasmáticos de lesão muscular AST, ALT, CK e LDH foram mensurados por métodos enzimáticos UV. Todas as análises bioquímicas plasmáticas foram realizadas em espectrofotômetro automatizado (BS-200 Chemistry Analyzer. Mindray. Hightech Industrial Park, Nanshan. China) a $37^{\circ} \mathrm{C}$, conforme orientações dos fabricantes, previamente ajustado com calibrador e soros controle níveis I e II comerciais (Biosystems, Barcelona, Spain).

Para avaliação do estresse oxidativo, foi quantificada a TAC pelo método inibição de formação de cátion de $\operatorname{ABTS}^{\circledR}$ (2.2'-Azino diethyl-bezothiazoline sulfonic acid), utilizandose conjunto de reativo comercial, conforme Erel (2004), assim como a concentração plasmática de albumina, bilirrubina e ácido úrico. A capacidade oxidante total plasmática foi determinada pelo método colorimétrico do xilenol laranja, conforme preconizado por Erel (2005). O teor plasmático de glutationa total (GSH) foi determinado utilizando-se conjunto de reagentes comerciais (Ransod and Ransel, Randox Laboratories, Oceanside, CA) e leitora automática de placas de 96 poços (Readwell Touch, Robonik PVT LTD, Thane, India), em $405 \mathrm{~nm}$, segundo recomendações do fabricante. A peroxidação lipídica plasmática foi determinada pelo método TBARS (substâncias reativas ao ácido tiobarbitúrico) utilizando-se reagente comercial (TBARS Assay Kit, ZeptoMetrix Corporation, USA) e leitora automática de placas de 96 poços (Readwell Touch, Robonik PVT LTD, Thane, India), em $545 \mathrm{~nm}$, de acordo com recomendações do fabricante. Os valores foram expressos em $\mu \mathrm{mol} / \mathrm{L}$ após comparação das amostras com uma curva de calibração com diversas concentrações de malondialdeído (MDA) proposta pelo fabricante.

Foram realizados estudos das distribuições das variáveis quanto à normalidade (teste $\mathrm{KS}$ ) e à homocedasticidade (teste Bartllet), conforme 
preconizado por Zar (1984). Para as comparações entre os quatro grupos experimentais das variáveis não paramétricas e paramétricas, foram utilizadas as provas de Kruskal-Wallis com pós-teste de Dunn e ANOVA com pós-teste de Tukey, respectivamente. As análises estatísticas supracitadas foram feitas com auxílio de um programa computacional estatístico, sendo considerado significativo $\mathrm{P}<0,05$.

\section{RESULTADOS E DISCUSSÃO}

Os resultados do perfil bioquímico plasmático dos ratos sedentários do grupo repouso e não tratado com RV (N-R-) ficaram dentro da faixa de referência da espécie(Melo et al., 2012; Dantas et al., 2006, Trall et al. 2004. O RV não alterou o perfil bioquímico plasmático dos ratos.

$\mathrm{O}$ esforço físico em ratos sedentários (N+R-) promoveu aumento da ureia e diminuição da glicose plasmática (Tab. 1). Comparados ao grupo controle (N-R-), os ratos sedentários submetidos ao esforço físico da natação (N+RV-) apresentaram sinais de lesão muscular, evidenciados pelo aumento significativo da atividade plasmática de $\mathrm{CK}$ e LDH, porém não das aminotransferases (Tab. 1).

No presente estudo, um único esforço físico em ratos sedentários (N+R-) causou estresse oxidativo caracterizado pelo aumento significativo de peroxidação lipídica do plasma e pela diminuição da TAC (Tab. 2). Não obstante a concentração de ácido úrico ter aumentado em ratos submetidos à natação $(\mathrm{N}+\mathrm{R}-)$, nesse grupo a TAC diminuiu (Tab. 2). Os 90min de natação em ratos induziram maior concentração de TBARS plasmático no grupo natação não tratado (N+R-) em relação ao controle (N-R-) (Tab. 2).

Ratos sedentários tratados com RV, quando submetidos a um único esforço físico súbito $(\mathrm{N}+\mathrm{R}+)$, apresentaram menor concentração de TBARS no plasma e aumento significativo da capacidade antioxidante total do plasma (Tab. 2). Não obstante a natação e o RV terem promovido uma elevação da TAC plasmática no grupo $\mathrm{N}+\mathrm{R}+$, nesse grupo foi observado aumento significativo de LDH e CK plasmático em relação ao grupo controle (N-R-) e menores concentrações de tais enzimas musculares em relação ao grupo N+R- (Tab. 1).

Tabela 1. Biomarcadores plasmáticos (média e desvio-padrão) de ratos Wistar sedentários submetidos ou não ao tratamento com resveratrol $(100 \mathrm{mg} / \mathrm{kg}$ PV/dia) por 14 dias e a 90 minutos de natação: N-R- (em repouso e não tratados com resveratrol); $\mathrm{N}-\mathrm{R}+$ (em repouso e tratados com resveratrol); $\mathrm{N}+\mathrm{R}$ - (natação e não tratados com resveratrol); $\mathrm{N}+\mathrm{R}+$ (natação e tratados com resveratrol)

\begin{tabular}{cccccc}
\hline Marcador & $\mathrm{N}-\mathrm{R}-$ & $\mathrm{N}-\mathrm{R}+$ & $\mathrm{N}+\mathrm{R}-$ & $\mathrm{N}+\mathrm{R}+$ & $\mathrm{P}$ valor \\
\hline Colesterol (mmol/L) & $1,92 \pm 0,21 \mathrm{a}$ & $1,58 \pm 0,28 \mathrm{ab}$ & $2,14 \pm 0,41 \mathrm{a}$ & $1,71 \pm 0,26 \mathrm{~b}$ & 0,0006 \\
Triglicérides(mmol/L) & $1,20 \pm 0,24 \mathrm{~b}$ & $1,07 \pm 0,27 \mathrm{~b}$ & $1,82 \pm 0,48 \mathrm{a}$ & $1,36 \pm 0,32 \mathrm{~b}$ & $<0,0001$ \\
AST (UI/L) & $110,41 \pm 7,76 \mathrm{a}$ & $122 \pm 25,65 \mathrm{a}$ & $133 \pm 30,65 \mathrm{a}$ & $122,25 \pm 18,85 \mathrm{a}$ & 0,1712 \\
ALT(UI/L) & $63,16 \pm 7,76 \mathrm{a}$ & $63,4 \pm 8,92 \mathrm{a}$ & $63,41 \pm 10,72 \mathrm{a}$ & $67,75 \pm 11,64 \mathrm{a}$ & 0,7067 \\
CK (UI/L) & $236,90 \pm 51,82 \mathrm{~b}$ & $275,7 \pm 106,15 \mathrm{~b}$ & $920,54 \pm 422,14 \mathrm{a}$ & $582,66 \pm 317,57 \mathrm{a}$ & $<0,0001$ \\
LDH(UI/L) & $227 \pm 82,31 \mathrm{c}$ & $289,2 \pm 138,99 \mathrm{bc}$ & $518,66 \pm 196,43 \mathrm{a}$ & $429,41 \pm 144,49 \mathrm{ab}$ & $<0,0001$ \\
\hline
\end{tabular}

*A presença de pelo menos uma letra coincidente na mesma linha indica que não há diferença estatística $(\mathrm{P}>0,05)$.

Tabela 2. Biomarcadores plasmáticos de estresse (média e desvio-padrão) de ratos Wistar sedentários submetidos ou não ao tratamento com resveratrol $(100 \mathrm{mg} / \mathrm{kg} \mathrm{PV} / \mathrm{dia})$ por 14 dias e a 90 minutos de natação: N-R- (em repouso e não tratados com resveratrol); N-R+ (em repouso e tratados com resveratrol); $\mathrm{N}+\mathrm{R}-$ (natação e não tratados com resveratrol); $\mathrm{N}+\mathrm{R}+$ (natação e tratados com resveratrol)

\begin{tabular}{cccccc}
\hline Marcador & $\mathrm{N}-\mathrm{R}-$ & $\mathrm{N}-\mathrm{R}+$ & $\mathrm{N}+\mathrm{R}-$ & $\mathrm{N}+\mathrm{R}+$ & $\mathrm{P}$ valor \\
\hline TAC $(\mathrm{mmol} / \mathrm{L})$ & $1,70 \pm 0,15 \mathrm{ab}$ & $1,64 \pm 0,23 \mathrm{~b}$ & $1,60 \pm 0,21 \mathrm{~b}$ & $1,89 \pm 0,11 \mathrm{a}$ & 0,0026 \\
Albumina $(\mathrm{g} / \mathrm{L})$ & $29 \pm 1,85 \mathrm{a}$ & $27,58 \pm 1,88 \mathrm{a}$ & $27 \pm 1,75 \mathrm{a}$ & $27,16 \pm 2,29 \mathrm{a}$ & 0,0650 \\
Bilirrubina total $(\mu \mathrm{mol} / \mathrm{L})$ & $12,56 \pm 3,21 \mathrm{a}$ & $13,02 \pm 4,46 \mathrm{a}$ & $15,87 \pm 5,90 \mathrm{a}$ & $11,54 \pm 4,95 \mathrm{a}$ & 0,1533 \\
Ácido úrico $(\mu \mathrm{mol} / \mathrm{L})$ & $108,55 \pm 8,59 \mathrm{~b}$ & $111,52 \pm 11,24 \mathrm{ab}$ & $124 \pm 13,15 \mathrm{a}$ & $105,03 \pm 5,90 \mathrm{~b}$ & 0,0012 \\
Glutationa $(\mathrm{nmol} / \mathrm{L})$ & $7,25 \pm 0,10 \mathrm{ab}$ & $7,10 \pm 0,15 \mathrm{~b}$ & $7,42 \pm 0,20 \mathrm{a}$ & $7,31 \pm 0,30 \mathrm{a}$ & 0,0025 \\
TBARS $(\mu \mathrm{mol} / \mathrm{L})$ & $14,85 \pm 8,15 \mathrm{bc}$ & $20,42 \pm 7,78 \mathrm{ab}$ & $37,36 \pm 16,75 \mathrm{a}$ & $5,85 \pm 3,15 \mathrm{c}$ & $<0,0001$ \\
\hline
\end{tabular}

*A presença de pelo menos uma letra coincidente na mesma linha indica que não há diferença estatística $(\mathrm{P}>0,05)$. 
As membranas celulares são ricas em ácidos graxos poli-insaturados, e o aumento do dano das células do músculo após o exercício físico intenso pode elevar a concentração de ácidos gordos poli-insaturados no sangue (Venditti; di Meo, 1996). O aumento do colesterol pode ser explicado pelo fato de o colesterol ser um constituinte importante e estar presente nas membranas celulares e o exercício físico provocar microlesões, segundo Marcondes et al., 1997. Além disso, a utilização de gordura como fonte de energia pode aumentar a biossíntese do colesterol (Bernardes et al., 2004), fazendo com que haja um aumento na sua concentração. Já os triglicerídeos se elevam no exercício físico devido à biogênese mitocondrial e ao aumento da expressão de transportadores e enzimas que regulam a oxidação de ácidos graxos no músculo esquelético, fato observado por Pinheiro et al. (2008). Quando a célula muscular é voltada para a oxidação, a gordura oxidada é utilizada pela mitocôndria para suprir as necessidades energéticas do exercício (Nadeau et al., 2006). Seu aumento pode estar relacionado com a hiperuricemia, dentre os quais uma das explicações seria que, durante a síntese de TG, haveria uma maior necessidade de NADPH para uma nova síntese de ácidos graxos (Oliveira et al., 2013).

Considerando-se que os ácidos graxos insaturados são suscetíveis à peroxidação lipídica, é provável que este seja um dos mecanismos pelo qual o exercício físico intenso aumenta a concentração de TBARS (Paschalis et al., 2007). O fato de os ratos sedentários tratados com RV submetidos a um esforço físico súbito $(\mathrm{N}+\mathrm{R}+)$ terem apresentado valores de colesterol, triglicérides significativamente menores pode explicar parcialmente a menor concentração de TBARS observada nesse grupo. Tal resultado sugere que o RV contribuiu para minimizar o estresse oxidativo causado pelo esforço físico súbito em ratos sedentários.

Os ratos sedentários submetidos ao esforço físico da natação (N+R-) apresentaram sinais de lesão muscular, evidenciado pelo aumento significativo da atividade plasmática de $\mathrm{CK}$ e LDH. O aumento da concentração de proteínas citosólicas na circulação após o exercício reflete a lesão muscular (Cruzat et al., 2007) e quando excede o limite da capacidade muscular, CK e LDH extravasa para o fluido intersticial e depois retorna para a circulação (Hammouda et al., 2012). Embora possa ocorrer um aumento detectável na atividade imediatamente após o exercício, o pico da CK é geralmente alcançado entre 24 e 72 horas pós-exercício (Cruzat et al., 2007). Esse aumento da atividade sérica de CK e LDH tem sido aceito como bom indicador de dano muscular (Foschini et al., 2008).

$\mathrm{O}$ ácido úrico, que é um produto final do metabolismo da purina, contribui para $58 \%$ da capacidade antioxidante do plasma (Trabelsi et al., 2011). Acredita-se que o ácido úrico aumente a fim de elevar a capacidade antioxidante total do plasma contra o estresse oxidativo e doenças inflamatórias moderadas (Oliveira et al., 2013). $\mathrm{O}$ aumento da concentração de ácido úrico observado nos ratos submetidos à natação $(\mathrm{N}+\mathrm{R}$ ) se deve provavelmente ao fato de o exercício intenso aumentar a degradação de purinas, principalmente no músculo esquelético, de modo a promover aumento da concentração de ácido úrico no plasma sanguíneo (Paschalis et al., 2007). Recentemente foi confirmado que o estresse oxidativo via ativação da xantina oxidase ocorre durante o exercício de alta intensidade e que o ácido úrico é um poderoso antioxidante (Hammouda et al., 2012).

Um único esforço físico (N+R-) em ratos sedentários causou estresse oxidativo com significativo aumento da peroxidação lipídica do plasma e diminuição da TAC. Portanto, o aumento do ácido úrico não foi suficiente para manter a TAC do plasma e evitar a lesão muscular. Os mecanismos envolvidos com o estresse oxidativo são complexos e acredita-se que um conjunto de alterações nos antioxidantes endógenos contribui para a atenuação desse estresse, eliminando ERO e radicais hidroxila (Clarkson; Thompson, 2000).

É sabido que o exercício físico intenso pode reduzir a TAC do organismo devido à excessiva produção de ERO (Fischer-Wellman; Bloomer, 2009) e que o treinamento físico regular promove uma adaptação do sistema antioxidante endógeno, minimizando o dano oxidativo. Esses resultados sugerem que os ratos sedentários são mais susceptíveis à lesão muscular e ao estresse oxidativo promovidos por um único esforço físico. 
Os noventa minutos de natação contínua em ratos sedentários foram capazes de aumentar a concentração de TBARS plasmático. O malondialdeído (MDA) estimado pela presença de TBARS é um biomarcador de estresse oxidativo cuja concentração depende da reação das ERO com os lipídeos das membranas celulares em resposta ao exercício físico (Urso; Clarkson, 2003). Segundo Dong et al. (2011), durante a atividade física intensa, a produção excessiva de ERO via NADPH-oxidase é provavelmente a principal razão para o elevado nível de peroxidação no sangue periférico.

Não obstante o efeito antioxidante do ácido úrico tenha diminuído em ratos tratados com $\mathrm{RV}$ e submetidos ao esforço físico $(\mathrm{N}+\mathrm{R}+)$, nesse grupo ocorreu um aumento significativo da TAC do plasma que pode ter contribuído para minimizar o estresse oxidativo. Esses resultados são concordantes com os de Fischer-Wellman; Bloomer (2009), que afirmam que, durante um esforço físico, o aumento de TAC é resultante da injúria pró-oxidante inicial e que o tratamento com antioxidante pode contribuir para esse aumento.

$\mathrm{O}$ aumento significativo de LDH e CK observado no grupo controle (N-R-) e as menores concentrações de tais enzimas musculares em relação ao grupo $\mathrm{N}+\mathrm{R}$ - sugerem que o RV na dose utilizada não foi capaz de evitar a lesão muscular em ratos sedentários submetidos a um único súbito esforço físico $(\mathrm{N}+\mathrm{R}+)$, porém conseguiu minimizá-la.

\section{CONCLUSÃO}

Resultados obtidos comprovam que animais sedentários submetidos ao esforço físico sofreram estresse oxidativo e lesão muscular, ainda que tais efeitos indesejáveis possam ser atenuados com o uso do antioxidante resveratrol.

\section{REFERÊNCIAS}

BAUR, J.A.; SINCLAIR, D.A. Therapeutic potential of resveratrol: the in vivo evidence. Nat. Rev. Drug Discovery, v.5, p.493-506, 2006.

BERNARDES, D.; MANZONI, M.S.J.; SOUZA, C.P. et al. Efeitos da dieta hiperlipídica e do treinamento de natação sobre o metabolismo de recuperação ao exercício em ratos. Rev. Bras. Educ. Fís. Esporte, v.18, p.191-200, 2004.
BOUSQUET-SANTOS, K.; VAISMAN, M.; BARRETO, N.D. et al. Resistance training improves muscle function and body composition in patients with hyperthyroidism. Arch. Phys. Med. Rehabil., v.87, p.1123-1130, 2006.

CLARKSON, P.M.; THOMPSON, H.S. Antioxidants: what role they play in physical activity and health? Am. J. Clin. Nutr., v.72, p.637-646, 2000.

CRUZAT, V.F.; ROGERO, M.M.; BORGES, M.C. et al. Current aspects about oxidative stress, physical exercise and supplementation. Rev. Bras. Med. Esporte, v.13, p.304-310, 2007.

DANTAS, J.A.; AMBIEL, C.R.; CUMAN, R.K.N. et al. Valores de referência de alguns parâmetros fisiológicos de ratos do Biotério Central da Universidade Estadual de Maringá, Estado do Paraná. Acta Sci. Health Sci., v.28, p.165-170, 2006.

DEATON, C.M.; MARLIN D.J. Exerciseassociated oxidative stress. Clin. Techn. Equine Pract., v.2, p.278-291, 2003.

DONG, J.; CHEN, P.; WANG, R. et al. NADPH oxidase: a target for the modulation of the excessive oxidase damage induced by overtraining in rat neutrophils. Int. J. Biol. Sci., v.7, p.881-891, 2011.

EREL, O. A new automated colorimetric method for measuring total oxidant status. Clin. Biochem., v.38, p.1103-1111, 2005.

EREL, O. A novel automated direct measurement method for total antioxidant capacity using a new generation, more stable ABTS radical cation. Clin. Biochem., v.37, p.277-285, 2004.

FISCHER-WELLMAN, K.; BLOOMER, R.J. Acute exercise and oxidative stress: a 30 year history. Dyn. Med., v.8, p.1-25, 2009.

FOSCHINI, D.; PRESTES, J.; LEITE, R.D. et al. Respostas hormonais, imunológicas e enzimáticas agudas a uma partida de basquetebol. Rev. Bras. Cineantropometria Desemp. Hum., v.10, p.341-346, 2008.

HAMMOUDA, O.; CHTOUROU, H.; CHAOUACHI, A. et al. Effect of Short-term maximal exercise on biochemical markers of muscle damage, total antioxidant status, and homocysteine levels in football players. Asian $J$. Sports Med., v.3, p.239-246, 2012. 
IKIZLER, M.; OVALI, C.; DERNEK, S. et al. Protective effects of resveratrol in ischemiareperfusion injury of skeletal muscle: a clinically relevant animal model for lower extremity ischemia. Chin. J. Physiol., v.49, p.204-209, 2006.

LEE, F.; KUO, T.; LIOU, S. et al. Chronic Rhodiola rosea extract supplementation enforces exhaustive swimming tolerance. Am. J. Chin. Med., v.37, p.557-72, 2009.

MARCONDES, M.C.C.G.; SIMÕES, G.C.; NEIVA, C.M. et al. Perfil lipídico de camundongos alimentados com dieta potencialmente aterogênica submetidos ao treinamento físico aeróbico. Rev. Bras. Ativ. Fis. Saúde, v.2, p.60-68, 1997.

MELO, M.G.D.; DÓRIA, A.A.; SERAFINI, M.R. et al. Valores de referência hematológicos e bioquímicos de ratos (Rattus novergicus linhagem Wistar) provenientes do biotério central da Universidade Federal de Sergipe. Sci. Plena, v.8, p.1-6, 2012.

NADEAU, K.J.; EHLERS, L.B.; AGUIRRE, L.E. et al. Exercise training and calorie restriction increase SREBP-1 expression and intramuscular triglyceride inskeletal muscle. Am. J. Physiol. Endocrinol. Metab., v.291, p.90-98, 2006.

NIE, J.; TONG, T.K.; GEORGE，K. et al. Resting and post exercise serum biomarkers of cardiac and skeletal muscle damage in adolescent runners. Scand. J. Med. Sci. Sports, v.20, p.1-5, 2010.

OLIVEIRA, E.P.; MORETO, F.; SILVEIRA, L.V.A. et al. Dietary, anthropometric, and biochemical determinants of uric acid in freeliving adults. Nutr. J., v.12, p.1-10, 2013.

PASCHALIS, V.; NIKOLAIDIS, M.G.; FATOUROS, I.G. et al. Uniform and prolonged changes in blood oxidative stress after muscledamaging exercise. In Vivo, v.21, p.877-884, 2007.

PINHEIRO, C.H.J.; SOUSA FILHO, W.M.; OLIVEIRA NETO, J. et al. Exercise prevents cardiometabolic alterations induced by chronic use of glucocorticoids. Arq. Bras. Cardiol., v.93, p.372-380, 2008.
PEARSON, K.J.; BAUR, J.A.; LEWIS, K.N. et al. Resveratrol delays age-related deterioration and mimics transcriptional aspects of dietary restriction without extending life span. Cell Metabolism, v.8, p.157-168, 2008.

POWERS, S.K.; JACKSON, M.J. Exerciseinduced oxidative stress: cellular mechanisms and impact on muscle force production. Physiol. Rev., v.88, p.1243-1276, 2008.

RYAN, M.J.; JACKSON, J.R.; HAO, Y. et al. Suppression of oxidative stress by resveratrol after isometric contractions in gastrocnemius muscles of aged mice. J. Gerontol. S. A Biol. Sci. Med. Sci., v.65, p.815-831, 2010.

SUN, M.; QIAN, F.; SHEN, W. et al. Mitochondrial nutrients stimulate performance and mitochondrial biogenesis in exhaustively exercised rats. Scand. J. Med. Sci. Sports, p.1-12, 2011.

THRALL, M.A.; BAKER, D.C.; LASSEN, E.D. et al. Veterinary hematology and clinical chemistry. Philadelphia: Lippincott Williams \& Wilkins, 2004. 516p.

TRABELSI, K.; EL ABED, K.; TREPANOWSKI, J.F. et al. Effects of ramadan fasting on biochemical and anthropometric parameters in physically active men. Asian $J$. Sports Med., v.2, p.134-144, 2011.

URSO, M.L.; CLARKSON, P.M. Oxidative stress, exercise, and antioxidant supplementation. Toxicology, v.189, p.41-54, 2003.

VENDITTI, P.; DI MEO, S. Antioxidants, tissue damage, and endurance in trained and untrained young male rats. Arch. Biochem. Biophys., v.331, p.63-8, 1996.

VESKOUKIS, A.S.; NIKOLAIDIS, M.G.; KYPAROS, A. et al. Blood reflects tissue oxidative stress depending on biomarker and tissue studied. Free Rad. Biol. Med., v.47, p.1371-1374, 2009.

ZAR, J.H. Biostatistical analysis. 2.ed. Washinton: Prentice Hall, 1984. p.718. 